\title{
Disordered graphene Josephson junctions
}

\author{
W. A. Muñoz, ${ }^{*}$ L. Covaci, ${ }^{\dagger}$ and F. M. Peeters ${ }^{\ddagger}$ \\ Departement Fysica, Universiteit Antwerpen, Groenenborgerlaan 171, B-2020 Antwerpen, Belgium
}

(Received 15 October 2014; revised manuscript received 22 January 2015; published 6 February 2015)

\begin{abstract}
A tight-binding approach based on the Chebyshev-Bogoliubov-de Gennes method is used to describe disordered single-layer graphene Josephson junctions. Scattering by vacancies, ripples, or charged impurities is included. We compute the Josephson current and investigate the nature of multiple Andreev reflections, which induce bound states appearing as peaks in the density of states for energies below the superconducting gap. In the presence of single-atom vacancies, we observe a strong suppression of the supercurrent, which is a consequence of strong intervalley scattering. Although lattice deformations should not induce intervalley scattering, we find that the supercurrent is still suppressed, which is due to the presence of pseudomagnetic barriers. For charged impurities, we consider two cases depending on whether the average doping is zero, i.e., existence of electron-hole puddles, or finite. In both cases, short-range impurities strongly affect the supercurrent, similar to the vacancies scenario.
\end{abstract}

DOI: 10.1103/PhysRevB.91.054506

PACS number(s): 73.43.-f, 73.23.-b, 73.63.-b

\section{INTRODUCTION}

The notable absence of intrinsic superconductivity in graphene has not been an obstacle for recent experimental advances demonstrating potential applications of graphene in superconducting devices by using the proximity effect [1-5]. Despite the fact that the interplay between superconductivity and quantum relativistic dynamics in graphene, expressed in an unusual Andreev reflection, has been elusive to experiments, advances in lithography make graphene-based superconducting devices a possible platform for superconducting quantum engineering. However, it has been observed that superconducting states in graphene are strongly affected by the inherent disorder that is present in graphene samples. More relevant, the specular Andreev reflection predicted to take place in a clean superconducting-normal $(\mathrm{S} / \mathrm{N})$ graphene interface, and where, different from the conventional retroreflection, the path of the reflected hole does not retrace the path of the incoming electron, cannot be observed in the presence of dopant inhomogeneities.

Many speculations have been made on the effect of disorder, like for instance the report of a gate-tunable Josephson junction where the off state at the Dirac point is believed to be caused by the suppression of the supercurrent due to intrinsic ripples appearing in graphene [4]. A suppression of the critical current due to the presence of puddles of charges has been reported as well [2]. Thus disorder can play an important role in graphene superconducting devices.

From a theoretical point of view, the interplay between superconductivity and disorder in graphene has not been thoroughly investigated. In only a few exceptional cases, works showing the role of disorder in intrinsic superconductivity $[6,7]$, as well as in $\mathrm{S} / \mathrm{N}$ graphene interfaces [8,9] have been recently reported. For instance, a counterintuitive enhancement of superconductivity by weak disorder has been predicted, while others have shown that the presence of disorder prevents the observation of the specular Andreev reflection and sup-

\footnotetext{
*williamarmando.munoz@uantwerp.be

†lucian@covaci.org

${ }^{\ddagger}$ francois.peeters@uantwerp.be
}

presses intrinsic superconductivity in graphene. From the perspective of the continuum Dirac approximation only scattering processes that mix the $K$ and $K^{\prime}$ valleys are predicted to matter [10]. However, it has been shown that inhomogeneous strain, which breaks the effective time-reversal symmetry in each cone but not the true time-reversal symmetry, can lead to the suppression of the Cooper diffusion in a graphene Josephson junction [11] by generating a pseudomagnetic barrier and allowing supercurrents to flow mainly as edge states.

The effect of disorder in graphene has been widely studied $[12,13]$ and was shown to break various symmetries, like the chiral or the effective time-reversal symmetries. The absence of these symmetries may strongly affect electronic transport [13-15]. The first and most commonly investigated type of extrinsic disorder corresponds to charge inhomogeneities $[14,16]$. This type of disorder resembles charged puddles, which are usually present when graphene is put on a substrate [17], or when, e.g., water molecules are deposited on its surface. Depending on the strength of the potential generated by these charged impurities, or the distance from the graphene sheet, they can be considered as long-range or short-range potentials. For instance, elastic scattering from a short-range disorder potential may mix electron states in different valleys $K$ and $K^{\prime}$, i.e., intervalley scattering. Instead, for a long-range potential varying smoothly over scales larger than the lattice constant, electrons in the $K$ and $K^{\prime}$ valleys do not mix [18].

Scattering on vacancies, i.e., the absence of a carbon atom $[14,19,20]$, unlike charged potentials, induces resonant states near the Dirac point. This short-range unitary scatterer may introduce a localized state, for which the wave function is formed equally from both $K$ and $K^{\prime}$ [19] valleys, similar to the nature of the wave function at armchair edges. For this type of disorder, coupling between valleys is expected to occur [21] and thus have a strong influence on the supercurrent.

Yet another type of disorder is induced by lattice distortions, either intrinsic or designed by strain engineering. Due to its exceptional flexibility, graphene can be easily deformed by mechanical stress or conform to the geometry of the substrate. Unusual high pseudomagnetic fields have been predicted to emerge from strained graphene [22]. In fact, theoretical descriptions have revealed the existence of an effective vector potential coming from the change in the hopping parameter 


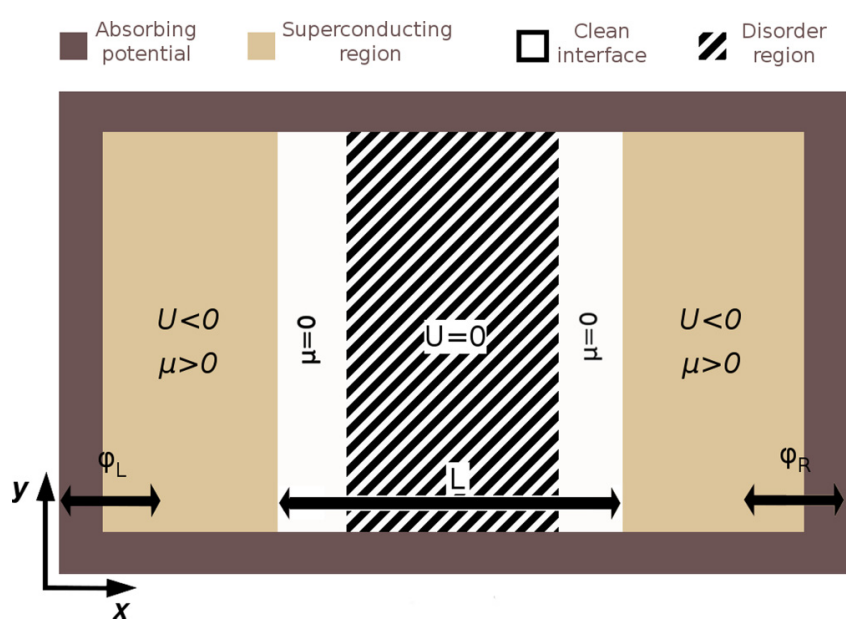

FIG. 1. (Color online) Layout of graphene Josephson junction considered in this work. Disordered and superconducting regions are separated by clean interface strips where Andreev reflection takes place.

due to the geometrical deformation of the distance between nearest-neighbor carbon atoms [23-27]. Particularly, some works have investigated the interplay between superconductivity and uniform strain [28] or pseudoquantum Hall states in graphene Josephson junctions [11].

Both electrons and holes experience normal scattering inside the junction, therefore different dephasing mechanisms are expected to strongly influence the transmission of the Copper pair between the superconducting leads. Since the more general description reported so far is based on the continuum Dirac approximation [10], a clear understanding of the effect of disorder in graphene Josephson junction is imperative. In this paper, we work directly at the tight-binding level and consider three different types of disorder: vacancies, ripples, and impurity scatterers. In all of these cases, we find that disorder affects the Andreev bound states that are formed in the junction. For instance, vacancies induce a zero-energy mode, which destroys the Andreev gap when the concentration of vacancies is increased. Similarly, short-range scatterers and strong pseudomagnetic fields will broaden and subsequently destroy the Andreev peaks. We calculate the Josephson current induced by the phase difference of the superconducting order parameter of the two contacts, and provide a qualitative picture on the effect of various types of disorder.

This paper is organized as follows. In Sec. II, we introduce our model and the numerical approach used. Results are organized according to the type of disorder considered. For instance, results concerning vacancies are presented and discussed in Sec. III, while the same is done for the cases regarding ripples and impurity scatterers in Secs. IV and V respectively. Finally, we summarize our findings in Sec. VI.

\section{MODEL}

A graphene Josephson junction is modeled according to the layout depicted in Fig. 1. Following closely the recipe implemented in previous works [29,30], we model the influence of the right and left superconducting contacts by assuming an on-site attractive pairing potential $U=-2.0 t_{0}$ and high doping, $\mu=0.6 t_{0}$, where $t_{0}=2.7 \mathrm{eV}$. In this way, we introduce a $s$-wave superconducting state over the outermost regions in the graphene sheet separated by a distance $L=$ $12.8 \mathrm{~nm}$. The width of the junction is considered to be much larger than the junction length, $W=73.8 \mathrm{~nm} \gg L$. We consider here an impurity-free $\mathrm{S} / \mathrm{N}$ interface, $l_{\text {int }}=1.5 \mathrm{~nm}$, seen in Fig. 1 as the white region with $\mu=0$ connecting the superconducting contacts and the disordered junction. We set a high Fermi level mismatch between the superconducting and the normal parts of the junction where the paring potential is set to zero $U=0$ (see Fig. 1). The large Fermi level mismatch between the highly doped contact region and the undoped interface may suppress Andreev reflection from nonrelativistic electrons [31]. We allow disorder only over the middle region of the junction, away from the interfaces, in order to guarantee that the leakage of the Coopers pairs is homogeneous along the clean interface strips. In addition, an absorbing region is imposed at the borders of the junctions in order to eliminate reflections coming from the boundaries, manifesting as finite size effects.

Finally, a dc-Josephson current is induced by fixing a phase difference $\Delta \phi=\phi_{R}-\phi_{L}$ between the outermost parts of the contact regions, as shown in Fig. 1. Because for the most configurations, the current-phase relation remains mostly unchanged, we keep the phase difference to be constant, $\Delta \phi=\pi / 2$. The calculation of the supercurrent is performed once the amplitude and the phase of the order parameter are relaxed in the superconducting region and convergence is achieved.

\section{A. The Chebyshev-Bogoliubov-de Gennes method}

The Andreev scattering process [32] in graphene is described within the Bogoliubov-de Gennes formalism by using the Nambu Gor'kov Green functions [33]:

$$
\mathcal{G}_{i j}=-\frac{i}{\hbar}\left(\begin{array}{cc}
\left\langle T c_{i \uparrow} c_{j \uparrow}^{\dagger}\right\rangle & \left\langle T c_{i \uparrow} c_{j \downarrow}\right\rangle \\
\left\langle T c_{i \downarrow}^{\dagger} c_{j \uparrow}^{\dagger}\right\rangle & \left\langle T c_{i \downarrow} c_{j \downarrow}^{\dagger}\right\rangle
\end{array}\right),
$$

where off-diagonal elements describe the amplitude of the superconducting order parameter coupling electrons and holes. Other physical quantities, such as the local density of states (LDOS), can be calculated from the Gor'kov Green's function corresponding to the diagonal elements of the matrix in Eq. (1), which is given by the expectation value of the inverse of the Hamiltonian: $\mathcal{G}=[\omega+i \eta-\mathcal{H}]^{-1}$.

The elements of the matrix (1) are calculated within the approximation of the Gor'kov Green's functions by implementing the Chebyshev-Bogoliubov-de Gennes method $[11,30,34]$ :

$$
G_{i j}^{1 \alpha}(\tilde{\omega})=\frac{-2 i}{\sqrt{1-\tilde{\omega}^{2}}}\left[\sum_{n=0}^{N} a_{i j}^{1 \alpha}(n) e^{-i n \arccos (\tilde{\omega})}\right],
$$

where the $N$-order expansion of the normal $(\alpha=1)$ and anomalous $(\alpha=2)$ coefficients corresponding to the diagonal and off-diagonal components of the Green's function [1] are defined accordingly as

$$
\begin{gathered}
a_{i j}^{11}(n)=\left\langle c_{i \uparrow}\left|T_{n}(\mathcal{H})\right| c_{j \uparrow}^{\dagger}\right\rangle, \\
a_{i j}^{12}(n)=\left\langle c_{i \downarrow}^{\dagger}\left|T_{n}(\mathcal{H})\right| c_{j \uparrow}^{\dagger}\right\rangle^{*},
\end{gathered}
$$


where $T_{n}(x)=\cos [n \arccos (x)]$ is the Chebyshev polynomial of the first kind of order $n$, which satisfies the following recurrence relation: $T_{n}(x)=2 x T_{n-1}(x)-T_{n-2}(x)$.

In the calculation of these moments, it is necessary to normalize the Hamiltonian matrix $\mathcal{H}$ and its eigenvalues $\omega$, according to $\tilde{\mathcal{H}}=(\mathcal{H}-\mathbb{1} b) / a$ and $\tilde{\omega}=(\omega-b) / a$, respectively, where the scale factors are $a=\left(E_{\max }-E_{\min }\right) /(2-\eta)$ and $b=\left(E_{\max }+E_{\min }\right) / 2$, with $\eta>0$ being a small number. Here, the extremal values $E_{\max }$ and $E_{\min }$ bound the energy spectrum of the BdG Hamiltonian, which in a real-space tight-binding formulation and at the mean-field level can been written following the Nambu notation (1):

$$
\mathcal{H}=\sum_{\langle i, j\rangle}\left(c_{i \uparrow}^{\dagger} c_{i \downarrow}\right) \hat{\mathcal{H}}_{i j}\left(\begin{array}{c}
c_{j \uparrow} \\
c_{j \downarrow}^{\dagger}
\end{array}\right),
$$

where the matrix $\mathcal{H}$ can be seen as arrangements of matrix blocks as follows:

$$
\hat{\mathcal{H}}_{i j}=\left(\begin{array}{cc}
\epsilon_{i}-\mu & \Delta_{i} \\
\Delta_{i}^{*} & \mu-\epsilon_{i}
\end{array}\right) \delta_{i j}+\left(\begin{array}{cc}
-t_{i j} & 0 \\
0 & t_{i j}^{*}
\end{array}\right)\left(1-\delta_{i j}\right),
$$

where $\epsilon_{i}$ denotes the on-site potential of the carbon atoms while $\mu$ is the chemical potential, which pins the Fermi energy. Nearest-neighbor $p_{z}$ orbitals $A_{i}$ and $B_{j}$ are coupled through the hopping parameter $t_{i j}$, which is known to be $t_{i j}=\gamma_{0} \sim 2.7 \mathrm{eV}$ for pristine graphene, where the minimum distance between carbon atoms is assumed to be $a_{0} \approx 1.42 \AA$. Inhomogeneous superconductivity is taken into account through the on-site $s$ wave order parameter $\Delta_{i}=U_{i}\left\langle c_{i \uparrow} c_{i \downarrow}\right\rangle$, where $U_{i}$ corresponds to the strength of the pairing potential and the complex correlation function $\left\langle c_{i \uparrow} c_{i \downarrow}\right\rangle$ is derived from the Gor'kov Green function (2) according to the following equation:

$$
\left\langle c_{i \uparrow} c_{i \downarrow}\right\rangle=\frac{i}{2 \pi} \int G_{i i}^{12}(\omega)[1-2 f(\omega)] d \omega,
$$

where $f(\omega)$ is the Fermi distribution function. Another physically relevant quantity is the local density of states, which is obtained from the Green's function (2) through the following formula:

$$
N^{i}(\omega)=-\frac{2}{\pi} \operatorname{Im} G_{i i}^{11}(\omega)
$$

\section{B. Calculation of the moments}

Once the Hamiltonian has been normalized, the expectation values in Eqs. (3) and (4), which define the expansion coefficients $a_{i j}^{1 \alpha}(n)=\left\langle\alpha \mid v_{n}\right\rangle$, where $\langle\alpha|$ are the vectors $\langle 1|=\left\langle c_{i \uparrow}\right|$ and $\langle 2|=\left\langle c_{i \downarrow}^{\dagger}\right|$, can be straightforwardly obtained through an iterative procedure involving a successive application of the Hamiltonian on the iterative vectors:

$$
a(n)=\left\langle\alpha \mid v_{n}\right\rangle=\left\langle\alpha \left|\left(2 \mathcal{H}\left|v_{n-1}\right\rangle-\left|v_{n-2}\right\rangle\right)\right.\right.
$$

with the initial conditions $\left|v_{0}\right\rangle=\left|c_{j \uparrow}\right\rangle$ and $\left|v_{1}\right\rangle=\mathcal{H}\left|v_{0}\right\rangle$. We refer the reader to Ref. [34] for more details about the method.

In order to obtain the average DOS in the disordered region, we use a more suitable approach to calculate the moments (3). It was shown that the average DOS can be expanded in terms of Chebyshev polynomials and the coefficients of order $n$ can be expressed as the trace of the polynomials of order $n$ of the Hamiltonian matrix. Instead of performing the full trace, i.e., averaging over the LDOS, we perform a stochastic evaluation of the trace of the Hamiltonian as follows [35]:

$$
a(n)=\operatorname{Tr}\left[T_{n}(\mathcal{H})\right] \approx \frac{1}{R} \sum_{r=0}^{R-1}\left\langle r\left|T_{n}(\mathcal{H})\right| r\right\rangle
$$

where the summation is carried out over $R$ random vectors $|r\rangle$, which in an arbitrary basis are defined as $|r\rangle=\xi_{r i}\left|c_{i \uparrow}^{\dagger}\right\rangle$ with random coefficients $\xi_{r i}$ having a normal distribution over the interval $[-1,1]$. The statistical average over the $R$ vectors of the expectation value approximates the trace in Eq. (10). Here the number of vectors $R$ required to perform (10) is much lower than the order $M$ of the Hamiltonian [36] and the number of atoms in the disordered region.

The average DOS is calculated from Eq. (8) with the moments obtained by using Eq. (10). The number of random vectors, $R$, is taken to be large enough such that the DOS converges. We typically use $R \sim 200$.

Since most of the computational effort comes from the sparse Hamiltonian matrix multiplications with iterative vectors, the performance is dramatically increased by implementing a parallel algorithm on graphical processing units (GPUs).

\section{Complex absorbing potential}

In order to mimic effectively an infinite region we introduce an absorbing potential operator $\hat{\Gamma}$ at the boundary by following the recipe of Ref. [37]. A proper choice of $\hat{\Gamma}$ requires its imaginary part to be negative $(\operatorname{Im} \hat{\Gamma} \leqslant 0)$ with an arbitrary choice for its real part, which we set to zero $(\operatorname{Re} \hat{\Gamma}=0)$ for convenience. It is expected that a well-behaved absorbing potential will eliminate reflection effects at the boundary. It can be shown that this absorbing boundary condition could be easily incorporated in the Chebyshev expansion of the Green's function by considering an imaginary damping factor $\hat{\gamma}$, which redefines the recursion formula (9) as follows:

$$
\left|v_{n}\right\rangle=e^{-\hat{\gamma}}\left(2 \mathcal{H}\left|v_{n-1}\right\rangle-e^{-\hat{\gamma}}\left|v_{n-2}\right\rangle\right),
$$

with the initial conditions $\left|v_{0}\right\rangle=\left|c_{i \uparrow}^{\dagger}\right\rangle$ and $\left|v_{1}\right\rangle=e^{-\hat{\gamma}}\left|v_{0}\right\rangle$. An energy-independent damping factor is given by $\hat{\gamma}(x)=$ $\sinh ^{-1}\{V(x)\}$ where absorbing potential $\hat{\Gamma}$ is defined as $\hat{\Gamma}(x)=$ $-i V(x)$. We consider here that the function $V(x)$ has a position dependence as it was deduced by Manolopoulos [38]:

$$
\hat{\Gamma}(x)=-i \alpha\left(\frac{2 \pi}{\Delta x}\right)^{2} f\left(x^{\prime}\right)
$$

where $\alpha$ is an universal constant while $\Delta x=x_{2}-x_{1}$ is the length of the absorbing region, and

$$
f\left(x^{\prime}\right)=\frac{4}{\left(c-x^{\prime}\right)^{2}}+\frac{4}{\left(c+x^{\prime}\right)^{2}}-\frac{8}{c^{2}}
$$

with $x^{\prime}=c\left(x-x_{1}\right) / \Delta x, c=2.62$ being a constant. Our calculations for pristine graphene (not presented here) show that we can remove all the finite size interference peaks in the LDOS, appearing due to scattering from the boundaries, without implementing periodic boundary conditions or considering very large lattice sizes. 


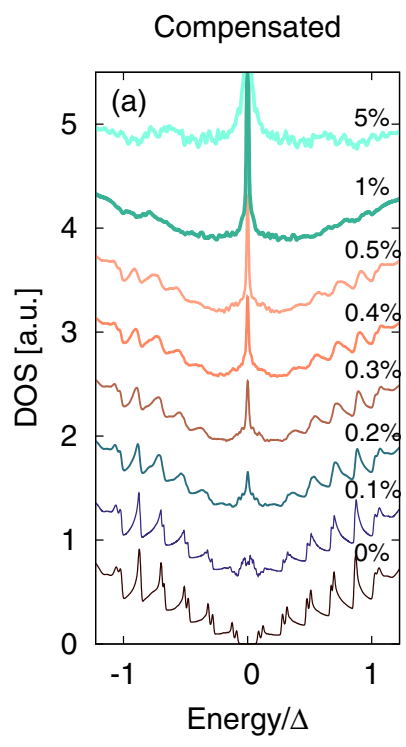

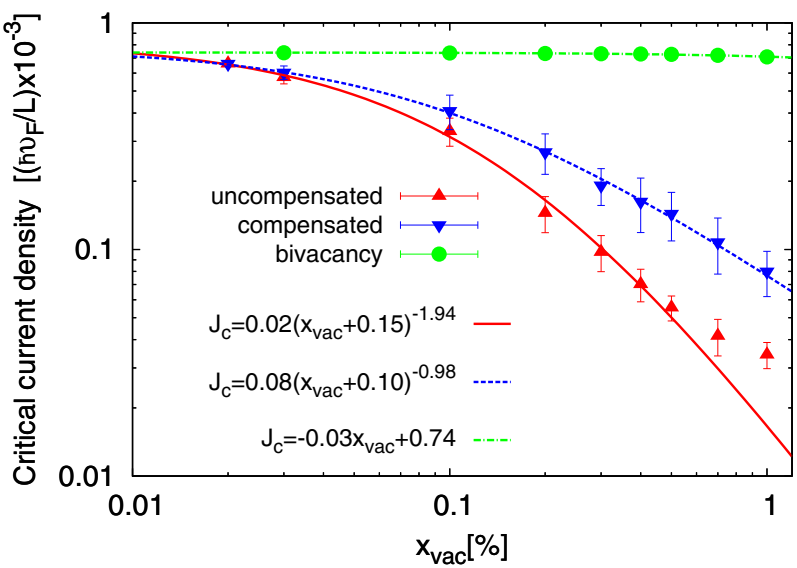

FIG. 3. (Color online) Average critical current density in a graphene Josephson junction for different concentrations of vacancies, diluted randomly over both sublattices (compensated), over a single sublattice (uncompesated), or over two-coupled sublattices (bivacancy). The continuous lines correspond to a least squares fit to $f(x)=a(x+b)^{c}$ and $g(x)=a x+b$.

FIG. 2. (Color online) DOS for a graphene Josephson junction considering different concentration of vacancies $x_{\mathrm{vac}}=N_{\mathrm{vac}} / N=$ $0,0.1 \%, 0.2 \%, 0.3 \%, 0.4 \%, 0.5 \%, 1 \%$, and $5 \%$ from bottom to top. Curves are progressively shifted in the vertical axis. Two cases are considered, according to the compensation in the distribution between the two sublattices: (a) completely compensated where vacancies are distributed equally in the two sublattices and (b) completely uncompensated where vacancies are randomly present only in one of the sublattices.

\section{RESULTS}

\section{A. Vacancies}

Within the tight-binding formalism considered here, a vacancy at atomic site $i$ is modeled by setting the on-site energy larger that any energy scale present in the pristine normal state in graphene, $\epsilon_{i} \gg 3 t$. In addition, the corresponding hopping parameters that connect the $i$ site to its neighbors are set to zero, $t_{i j}=0$. Our numerical procedure considers a finite concentration of vacancies, defined through the ratio $x_{\mathrm{vac}}=N_{\mathrm{vac}} / N$, where $N$ is the total number of atomic sites in the junction.

It has been shown that divergences in the DOS are present around the Dirac point when a finite concentration of vacancies are induced in graphene [19]. Here, we calculate the DOS of the graphene Josephson junction while introducing different concentrations of vacancies over the disordered region depicted in Fig. 1. The DOS is averaged over different realizations for the configuration of vacancies in order to include all possible scattering and interference processes between the electron/hole wave and the scatterers induced by the missing atoms. In Fig. 2, we shown the different Andreev peaks that are present in our clean Josephson junction of graphene (bottom curve). The electron and hole waves are scattered by the $\mathrm{N} / \mathrm{S}$ interface and interfere, giving rise to the well-known Andreev peaks. Note that because the chemical potential in the junction is at the Dirac point, the Andreev peaks show the specific energy dependence in the graphene junctions, i.e., traveling modes with a gapped spectrum versus bound states with an ungapped spectrum as observed in conventional S/N/S junctions. As we can see clearly in Fig. 2, as the concentration of impurities increases, the Andreev peaks are progressively suppressed. Notice that the lowest energy peaks are the first to disappear as the scattering probability for long paths between the interfaces becomes higher, affecting lower energy states, when compared to short paths, which contribute to higher subgap energy states. As the concentration is increased above $x>0.5 \%$, traces of the Andreev reflection processes on the DOS vanish.

The selective dilution of the vacancies in the different sublattice sites has been shown to induce different zero modes in the DOS [19,39]. For instance, complete dilution of the vacancies in one of the sublattices, or complete uncompensated dilution, leads to the opening of a gap around the zero mode at the Dirac point and whose magnitude is proportional to the vacancy concentration. On the contrary, complete compensated dilution brings an increase of the spectral weight for energies surrounding the Dirac point. We have investigated both cases and found slight differences in the DOS around the Fermi level, which are more remarkable for larger vacancy concentrations, $x>0.5 \%$ (see Fig. 2). In order to further clarify the contrast between the compensated and uncompensated cases we show in Fig. 3 the average critical current density across the junction as a function of vacancy concentration. Note that the current is averaged over the junction width and over impurity configurations. As we can observe, both cases lead to different power-law suppression of the critical current. For instance, the suppression of the current goes according to $J_{c} \sim x^{-1.8}$ for uncompensated dilutions of the vacancy configurations. This agrees with the fact that a gap of energy scale $E^{2} \sim x_{\text {vac }}$ is induced for complete uncompensated dilution of vacancies in graphene [19]. On the other hand, vacancies diluted in both sublattices still show a strong suppression of the supercurrent but weaker than the uncompensated case. As an interesting fact, we note that by placing these vacancies in pairs of bounded atomic sites (bivacancies), we can observed that the suppression is much 
weaker than in previous cases. The slow linear suppression of the supercurrent in the presence of bivacancies is due to the absence of intervalley scattering, as was already reported previously for this type of atomic-scale defects [21].

\section{B. Ripples: Gaussian bumps}

We next analyze the effects of inhomogeneous strain over the disordered area in the graphene Josephson junction. For this purpose, we model the ripples in graphene as smooth bumps where the out of plane deformation is described by a Gaussian function. It has been theoretically shown that sixfold spatially symmetric pseudomagnetic fields, with alternating sign, emerge from this strain configuration [27,40,41]. The Gaussian deformation is introduced in the tight-binding description of Eq. (6) by the strained hopping parameter:

$$
t_{i j}=\gamma_{0} \exp ^{-3.37\left(\frac{l_{i j}}{a_{0}}-1\right)},
$$

where $\gamma_{0}=2.7 \mathrm{eV}$ and $a_{0}=1.42 \AA$ are the unstrained hopping and lattice parameter, respectively, while $l_{i j}$ is the strained distance between nearest neighbors $i$ and $j$. The corresponding out of plane deformation is given by a Gaussian function as follows:

$$
Z\left(R_{i j}\right)=Z_{0} \exp ^{-\left|R_{i j}\right|^{2} / 2 \varepsilon^{2}},
$$

where $R_{i j}=r_{i j}-R_{0}$ is the in-plane atomic position with respect to the center of the Gaussian, $R_{0}$. It is important to mention that the Gaussian width parameter $\varepsilon$ is constrained here, such that $Z\left(R_{i j}\right) \approx 0$ in the clean interface regions (see Fig. 1). Once the width is fixed, the height parameter $Z_{0}$ is adjusted according to a desired maximal strain. Since we known from the continuum model how the strength of the pseudomagnetic field depends on the parameters of the Gaussian, we considered different configurations for the size and the number of Gaussian bumps inside the junction (see Fig. 4). As a particular case, we have included in Fig. 4(d) an arrangement of triangular bumps composed by a superposition of four Gaussians, where three are centered at the vertices of an equilateral triangle, while the fourth one is placed at the circumcenter of the triangle. This particular strain has been inspired by a previous theoretical study where a nonuniform deformation is engineered by depositing graphene on a substrate decorated with nanopillars set in a triangular configuration [42]. The corresponding pseudomagnetic fields emerging from this deformation exhibit a nontrivial symmetry, consisting of larger regions with an almost constant pseudomagnetic field, when compared to the sixfold symmetric fields generated by isolated Gaussian bumps. In order to investigate the effect of ripples on Andreev scattering in the junction we calculated the average DOS for the different configurations of Gaussian bumps shown in Fig. 4. These results are shown in Figs. 5(a)-5(d) for different values of the maximum strain: 0\%, $5 \%, 10 \%$, and $20 \%$. The Andreev states seen in Fig. 5(a) start to be affected by the presence of the Gaussian bumps even for the lowest values of the strain. Particularly, high-energy Andreev peaks are mainly suppressed in the case of a higher density of Gaussian bumps depicted in Fig. 4(a). This suggests that short paths are influenced more by the pseudomagnetic field, rather than the long paths that contribute to the low-energy spectrum.

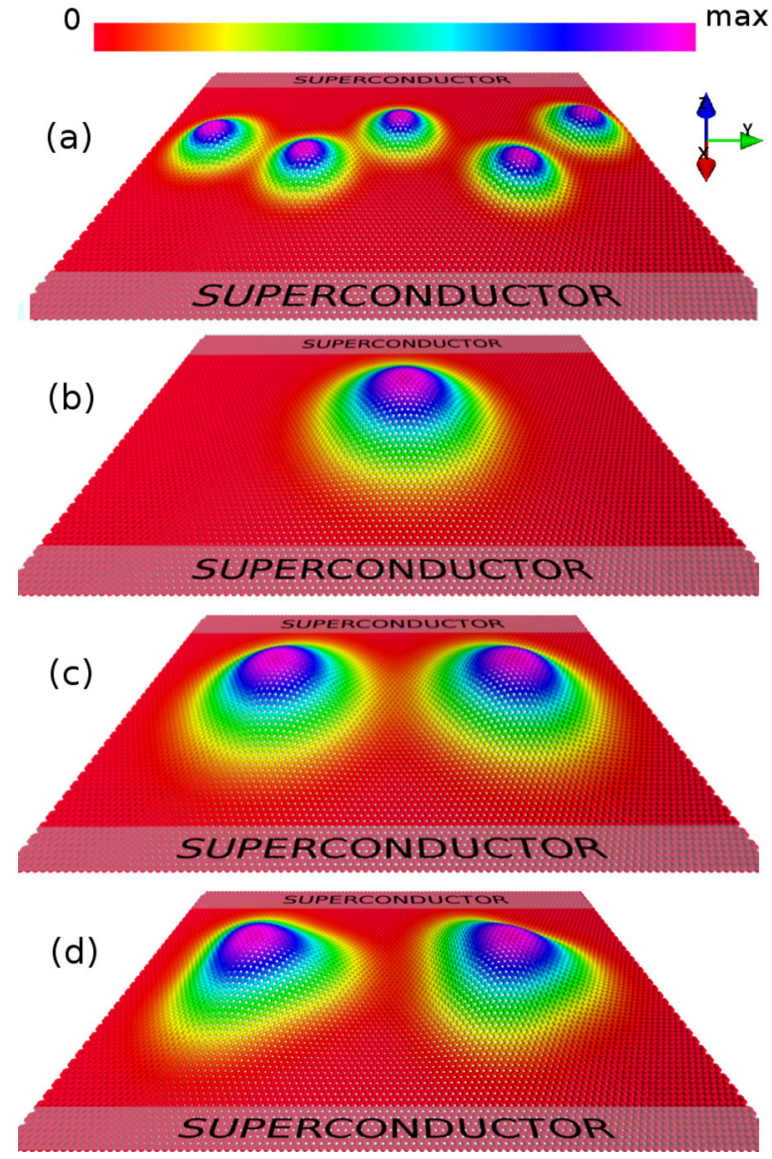

FIG. 4. (Color online) Strained graphene Josephson junction with different configurations of Gaussian bumps. (a)-(c) show isolated Gaussian bumps, while (d) corresponds to an arrangement of four Gaussian bumps in a triangular configuration.

Alternatively, the configuration with a single large Gaussian bump, shown in Fig. 4(b), for which the DOS is presented in Fig. 5(b) shows that high-energy Andreev peaks remain conserved despite the fact that the pseudomagnetic field is supposed to be stronger over a wider region. At low energies, sharp peaks appear, and the quasiparticle gap starts to close.

The next two cases, shown in Figs. 4(c), 4(d), 5(c), and $5(d)$, reveal an interplay between the size of the Gaussian bump and the strength of the pesudomagnetic field. As seen previously, the high energy peaks are the first to be affected as the strain is increased. At low energies, Landau level-like peaks appear and the gap observed for the clean system closes. Because of the oscillating pseudomagnetic field, a combination of snakelike states and pseudo-Landau levels, appear where the field vanishes or is maximal. The existence of extended regions with large pseudomagnetic fields, will act as a pseudomagnetic barrier for the electron or hole quasiparticles propagating in the junction. As a consequence, although the time-reversal symmetry is not broken, it is expected that the supercurrent is suppressed and will flow mainly as edge states near the boundaries [11]. We next investigate the Josephson current flowing through the strained junctions in Fig. 6, where we present the average critical current density as a function of the maximum strain applied in each configuration. We find a 

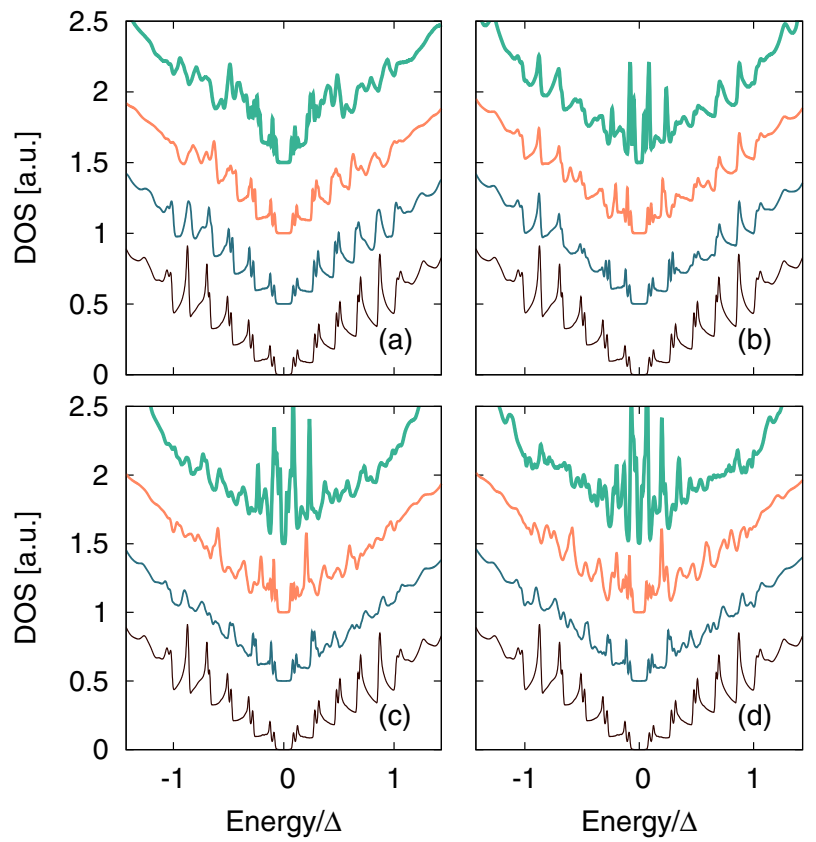

FIG. 5. (Color online) DOS for a graphene Josephson junction for the different strain configurations shown in Fig. 4. Panels from left to right correspond to cases from top to bottom shown in Fig. 4, respectively. Andreev peaks are shown for deformations with the maximum strain increasing from $0 \%$ to $5 \%, 10 \%$, and $20 \%$ (bottom to top). Curves are progressively shifted in the vertical axis inside each panel.

suppression of the critical current as the strain is increased. In particular, this suppression is stronger for the configurations shown in Figs. 4(c) and 4(d) when compared with the single bump case, Fig. 4(b), and the many smaller bumps, Fig. 4(a). As suggested previously, these results can be explained by noticing that the current is more effectively suppressed in the cases in which the pseudomagnetic field is larger over a more extended area, thus providing a more efficient magnetic barrier. Thus we can infer that the number of bumps is not as important as the effective area covered by a single bump. Although the

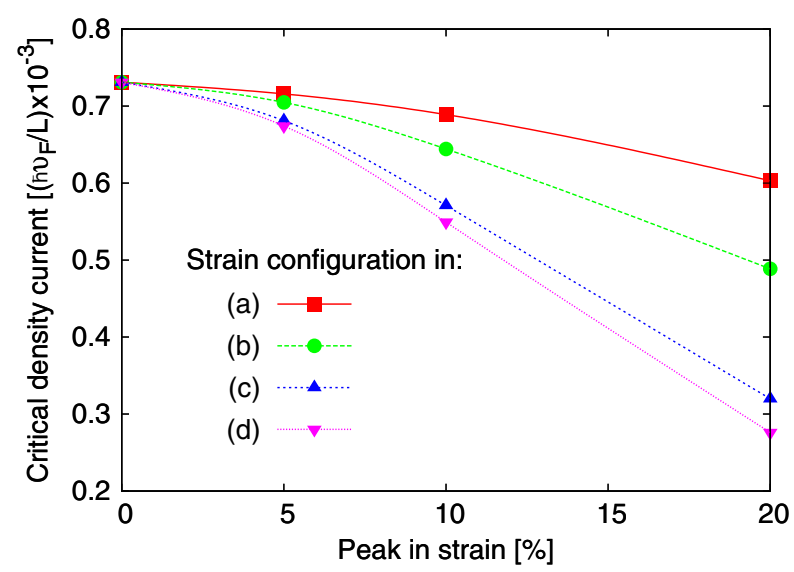

FIG. 6. (Color online) Average critical current density in strained Josephson junctions as a function of maximal strain for the strain configurations depicted in Figs. 4(a)-4(d). size of the bump in case (d) is the same as the one in case (c), the current is further suppressed because the triangular bump induces larger regions with pseudomagnetic fields with the same sign, although the average field is zero.

\section{Charged impurities}

Finally, we investigate the effect of scattering due to the presence of impurities on the diffusion of Andreev pair in graphene Josephson junctions. It is well known that the presence of these types of impurities may induce local charged puddles in graphene, which can be emulated through spatial fluctuations of the Fermi energy around the Dirac point. These fluctuations are modeled here by means of a random superposition of $N_{\text {imp }}$ potentials with a Gaussian-like spatial dependence. Thus, we assume that the proximity of a single charged impurity is reflected in the parameters of a Gaussian potential such that on-site energies of carbon atoms in the disordered region are given as follows [43]:

$$
\epsilon_{i}=\sum_{j=1}^{N_{\text {imp }}} V_{j} \exp \left(-\frac{\left|r_{i}-R_{j}\right|^{2}}{2 \varepsilon^{2}}\right),
$$

where $V_{j}$ and $\varepsilon$ correspond to the amplitude and range of a single Gaussian potential centered at the atomic position $R_{j}$, respectively. These on-site potentials as described by Eq. (16) are introduced in our formalism described by the Hamiltonian (6) but we constrain their scope to $\varepsilon<L, W$ such that they vanish in the interface strips where the Fermi level is pinned at the Dirac point.

In order to characterize the effect of this sort of disorder, we follow the recipe proposed in previous works $[43,44]$ where the mean-free path is considered as being inversely proportional to the following parameter:

$$
\kappa_{0} \propto\left(\frac{V_{i}}{t}\right)^{2} x_{\mathrm{imp}} \kappa^{2},
$$

where $V_{i} / t$ is the amplitude of the random potential in units of the hopping parameter, while the ratio of the number of impurities and the total number of atoms in the junction corresponds to the concentration of impurities $x_{\text {imp }}=N_{\text {imp }} / N$. The averaged charge density per impurity atom is described by the factor $\kappa$ according to

$$
\kappa=\frac{1}{N_{\text {imp }}} \sum_{j}^{N_{\text {imp }}} \sum_{i}^{N} \exp \left(-\frac{\left|x_{i}-x_{j}\right|^{2}}{2 \varepsilon^{2}}\right) .
$$

For practical purposes, we consider the same amplitude for all potentials in Eq. (16), i.e., $\left|V_{j}\right|=V$ for all $j$. In addition, for a given value of the Gaussian width $\varepsilon$, we fixed the maximum of the Gaussian, $V$, such that the density of charges obtained from Eq. (18) is the same for the different values of $\varepsilon$ considered here. This allows us to make a clearer discussion about the effect of the Gaussian potential, mainly of its height and width parameters, as long as the total charge density is kept fixed under a constant concentration of vacancies.

We consider two separate cases. First, in the presence of electron and hole puddles, the total charge density is zero, meaning that the number of electron and hole-doped Gaussians is equal. We next investigate the presence of charged impurites 

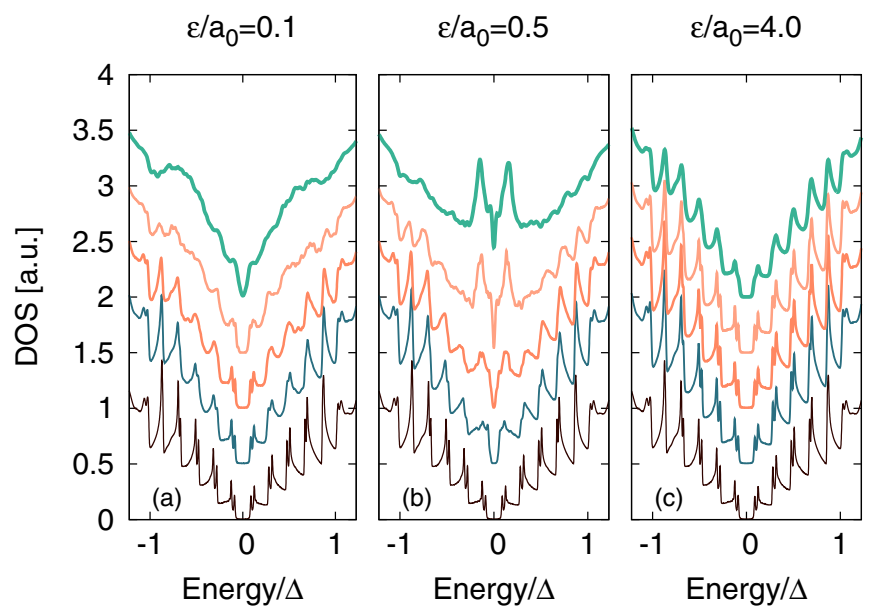

FIG. 7. (Color online) Average DOS for disordered graphene Josephson junctions, considering impurity scattering potentials with different ranges, $\varepsilon / a_{0}=0.1,0.5$, and 4.0. Different values of the impurity concentration considered inside the panels are $x_{\text {imp }}=0 \%$, $1 \%, 2 \%, 3 \%$, and $5 \%$, from bottom to top, where the curves are progressively shifted in the vertical axis inside each panel.

of the same type, i.e., electron-doped Gaussians, in which case the charge density will increase as the concentration of impurities increases.

We first present our results for the electron-hole charge puddles. In Figs. 7(a)-7(c), we show the average DOS in a graphene Josephson junction with doping inhomogeneity profiles given by Eq. (16) for three different cases according to the size of the Gaussian potential induced by single impurities. As in the previous disorder cases, the DOS is averaged over different realizations of the impurity configurations. The most trivial case, where $\varepsilon / a_{0}=0.1 \ll 1$, is depicted in Fig. 7(a). In this limit, which resembles the typical Anderson disorder model, we can observe that the effect of increasing the concentration of impurities $x_{\text {imp }}$ leads to a suppression of the Andreev bound states. Despite the fact that this on-site defect is similar to the case of vacancies (in the limit of large $V$ ) we can see that when comparing with Fig. 2 the dispersion mechanism acts differently in the two cases, as lower energy Andreev states are preserved even for the higher values of $x_{\text {vac }}$ in Fig. 7(a).

Next, we proceed to a larger $\varepsilon / a_{0}=0.5$, a value still smaller than the lattice parameter. In this situation, we see in Fig. 7(b) that Andreev bound states are affected much more strongly as intervalley scattering is expected to become more pronounced. It is clearly seen that the lower energy gap disappears in the first place as the concentration of impurities is increased. This is similar to what was observed for vacancies, with the difference that localization peaks appear at finite energy instead of Fermi energy. We confirmed that the low energy peaks observed in Fig. 7(b) for large impurity concentration are not Andreev peaks because the appear also for a nonsuperconducting system. They signal the appearance of strongly localized state, which will shift towards the Fermi energy as $V$ is increased.

Contrary to the previous cases, when $\varepsilon>a_{0}$, the influence of disorder on the Andreev states is weak. As the potential profile becomes smoother on the scale of the lattice parameter,
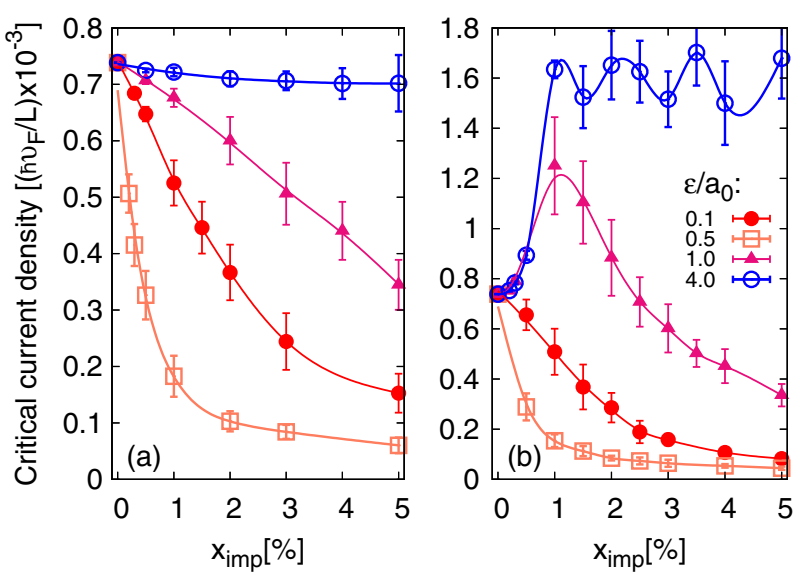

FIG. 8. (Color online) Average critical current density in a graphene Josephson junction as a function of the impurity concentration $x_{\text {imp }}$ for different widths of the Gaussian potential induced by isolated impurities. Equal number of electron and holelike potentials are considered in (a), while only electronlike potentials are assumed in (b) leading to an inhomogeneous but finitely doped junction.

intervalley scattering is suppressed, thus having a weak influence on the average DOS in the junction, as seen in Fig. 7(c). In order to verify the insights given by the change in the Andreev levels seen in the averaged DOS, we plot in Fig. 8 the average critical current density as a function of impurity concentration for different values of $\varepsilon$. First, in panel Fig. 8(a), the electron-hole puddles scenario is considered. In this case, the current is suppressed for all ranges of the potential profiles, with a much stronger effect when $\varepsilon \leqslant a_{0}$, and a very weak effect when $\varepsilon>a_{0}$. The case $\varepsilon / a_{0}=0.5$ deserves particular attention as the current becomes strongly suppressed when the impurity concentration is increased, confirming the strong suppression of the Andreev peaks observer in the averaged DOS. The effect of charge puddles was investigated experimentally in graphene Josephson junctions in the longjunction limit [2], and the presence of these puddles was given as an explanation for the strong suppression of the supercurrent at charge neutrality. As shown here, we only observe a strong suppression when the charge scatterers are short-ranged.

In addition to the charge puddles case, the effect of an impurity distribution with positive charge, inducing an average finite doping in graphene, is also studied. The average critical current density for this scenario is presented in Fig. 8(b). Here the dependence of the current is not monotonic as a function of $\varepsilon$. The overall tendency is for the supercurrent to be enhanced since finite doping brings the Fermi level away from the Dirac point. On the other hand, the presence of short-range scatterers will also generate intervalley scattering events, thus suppressing the current. We therefore observe two separate regimes, depending on whether $\varepsilon<a_{0}$ or $\varepsilon \geqslant a_{0}$. For $\varepsilon=$ $0.1 a_{0}$ and $0.5 a_{0}$, the current is suppressed even for low impurity concentration, signaling the fact that intervalley scattering is the main contribution, larger for $\varepsilon=0.5 a_{0}$ due to stronger localization effects. If $\varepsilon \geqslant a_{0}$, at low impurity concentration the supercurrent is increasing due to an increase in the average doping but depending on the intervalley scattering probability it will be eventually suppressed, more for $\varepsilon=a_{0}$ than $\varepsilon=4 a_{0}$. 


\section{CONCLUSION}

In conclusion, by using a numerical tight-binding approach, we described various disorder scenarios in graphene Josephson junctions near charge neutrality. We investigated both the disappearance of the multiple Andreev reflection peaks in the junction and the suppression of the Josephson current. We observed that the supercurrent is most strongly suppressed in the presence of vacancies or resonant impurities, e.g., adsorbed hydrogen atoms. In this case, the presence of strong intervalley scattering destroys the interference of time reversed electron-hole pairs, which undergo Andreev reflections at the $\mathrm{N} / \mathrm{S}$ interfaces. As a test, we show that when the vacancies come in pairs, and thus the sublattice symmetry is not being broken, the supercurrent is very weakly suppressed.

Another scattering mechanism is given by the presence of ripples. We show that although there should be no intervalley scattering in this case, Gaussian bumps will act as pseudomagnetic barriers, thus suppressing the supercurrent.
The larger the regions with finite pseudomagnetic fields, the more efficient the barriers will be.

A third disorder scenario involves the presence of charged impurities, which are modeled as variations of the local potential. We show that in the presence of electron-hole charge puddles, the supercurrent is always suppressed, but the strongest effect is obtained when the range of the potential disorder is very small, thus inducing significant intervalley scattering. When the impurities only dope with electrons, we observe an interplay between an enhancement of the current due to the shift of the Fermi energy away from the Dirac point, and a suppression by short-range scatterers due to intervalley scattering.

\section{ACKNOWLEDGMENT}

This work was supported by the Flemish Science Foundation (FWO-Vlaanderen) and the Methusalem funding of the Flemish Government.
[1] H. B. Heersche, P. Jarillo-Herrero, J. B. Oostinga, L. M. K. Vandersypen, and A. F. Morpurgo, Nature (London) 446, 56 (2007).

[2] K. Komatsu, C. Li, S. Autier-Laurent, H. Bouchiat, and S. Guéron, Phys. Rev. B 86, 115412 (2012).

[3] N. Mizuno, B. Nielsen, and X. Du, Nat. Commun. 4, 2716 (2013).

[4] J.-H. Choi, G.-H. Lee, S. Park, D. Jeong, J.-O. Lee, H.-S. Sim, Y.-J. Doh, and H.-J. Lee, Nat. Commun. 4, 2525 (2013).

[5] F. Deon, S. Šopić, and A. F. Morpurgo, Phys. Rev. Lett. 112, 126803 (2014).

[6] R. Nandkishore, J. Maciejko, D. A. Huse, and S. L. Sondhi, Phys. Rev. B 87, 174511 (2013).

[7] I.-D. Potirniche, J. Maciejko, R. Nandkishore, and S. L. Sondhi, Phys. Rev. B 90, 094516 (2014).

[8] S.-g. Cheng, H. Zhang, and Q.-f. Sun, Phys. Rev. B 83, 235403 (2011).

[9] P. Burset, W. Herrera, and A. Levy Yeyati, Phys. Rev. B 80, 041402 (2009).

[10] C. W. J. Beenakker, Rev. Mod. Phys. 80, 1337 (2008).

[11] L. Covaci and F. M. Peeters, Phys. Rev. B 84, 241401 (2011).

[12] A. H. Castro Neto, F. Guinea, N. M. R. Peres, K. S. Novoselov, and A. K. Geim, Rev. Mod. Phys. 81, 109 (2009).

[13] E. R. Mucciolo and C. H. Lewenkopf, J. Phys. Condens. Matter 22, 273201 (2010).

[14] N. M. R. Peres, F. Guinea, and A. H. Castro Neto, Phys. Rev. B 73, 125411 (2006).

[15] A. F. Morpurgo and F. Guinea, Phys. Rev. Lett. 97, 196804 (2006).

[16] T. Ando, J. Phys. Soc. Jpn. 75, 074716 (2006).

[17] J.-H. Chen, C. Jang, S. Adam, M. S. Fuhrer, E. D. Williams, and M. Ishigami, Nat. Phys. 4, 377 (2008).

[18] T. Ando and T. Nakanishi, J. Phys. Soc. Jpn. 67, 1704 (1998).

[19] V. M. Pereira, F. Guinea, J. M. B. Lopes dos Santos, N. M. R. Peres, and A. H. Castro Neto, Phys. Rev. Lett. 96, 036801 (2006).
[20] V. M. Pereira, J. M. B. Lopes dos Santos, and A. H. Castro Neto, Phys. Rev. B 77, 115109 (2008).

[21] C. Park, H. Yang, A. J. Mayne, G. Dujardin, S. Seo, Y. Kuk, J. Ihm, and G. Kim, PNAS 108, 18622 (2011).

[22] N. Levy, S. A. Burke, K. L. Meaker, M. Panlasigui, A. Zettl, F. Guinea, A. H. C. Neto, and M. F. Crommie, Science 329, 544 (2010).

[23] F. de Juan, A. Cortijo, and M. A. H. Vozmediano, Phys. Rev. B 76, 165409 (2007).

[24] F. Guinea, M. I. Katsnelson, and A. K. Geim, Nat. Phys. 6, 30 (2010).

[25] V. M. Pereira and A. H. Castro Neto, Phys. Rev. Lett. 103, 046801 (2009).

[26] F. Guinea, A. K. Geim, M. I. Katsnelson, and K. S. Novoselov, Phys. Rev. B 81, 035408 (2010).

[27] D. Moldovan, M. Ramezani Masir, and F. M. Peeters, Phys. Rev. B 88, 035446 (2013).

[28] M. Alidoust and J. Linder, Phys. Rev. B 84, 035407 (2011).

[29] A. M. Black-Schaffer and S. Doniach, Phys. Rev. B 78, 024504 (2008).

[30] W. A. Muñoz, L. Covaci, and F. M. Peeters, Phys. Rev. B 86, 184505 (2012).

[31] A. R. Akhmerov and C. W. J. Beenakker, Phys. Rev. B 75, 045426 (2007).

[32] A. Andreev, Sov. Phys. JETP 19, 1228 (1964).

[33] Y. Nambu, Phys. Rev. 117, 648 (1960).

[34] L. Covaci, F. M. Peeters, and M. Berciu, Phys. Rev. Lett. 105, 167006 (2010).

[35] A. Weiße, G. Wellein, A. Alvermann, and H. Fehske, Rev. Mod. Phys. 78, 275 (2006).

[36] T. Iitaka and T. Ebisuzaki, Phys. Rev. E 69, 057701 (2004).

[37] V. A. Mandelshtam and H. S. Taylor, J. Chem. Phys. 103, 2903 (1995).

[38] D. E. Manolopoulos, J. Chem. Phys. 117, 9552 (2002). 
[39] A. Cresti, F. Ortmann, T. Louvet, D. Van Tuan, and S. Roche, Phys. Rev. Lett. 110, 196601 (2013).

[40] G. M. M. Wakker, R. P. Tiwari, and M. Blaauboer, Phys. Rev. B 84, 195427 (2011).

[41] N. N. Klimov, S. Jung, S. Zhu, T. Li, C. A. Wright, S. D. Solares, D. B. Newell, N. B. Zhitenev, and J. A. Stroscio, Science 336, 1557 (2012).
[42] M. Neek-Amal, L. Covaci, and F. M. Peeters, Phys. Rev. B 86, 041405 (2012).

[43] A. Rycerz, J. Tworzydło, and C. W. J. Beenakker, Europhys. Lett. 79, 57003 (2007).

[44] C. H. Lewenkopf, E. R. Mucciolo, and A. H. Castro Neto, Phys. Rev. B 77, 081410 (2008). 